Bull. Austral. Math. Soc.

VOL. 53 (1996) [101-107]

\title{
ON THE SELF-LENGTH OF TWO-DIMENSIONAL BANACH SPACES
}

\author{
B. Chalmers, C. Franchetti and M. Glaquinta
}

The aim of this paper is to prove the following result: if $X$ is a 2-dimensional symmetric real Banach space, then its self-length is greater than or equal to $2 \pi$. Moreover, the minimum value $2 \pi$ is uniquely attained (up to isometries) by euclidean space.

\section{SYMMETRY NOTIONS AND PROJECTION CONSTANTS}

An $n$-dimensional real Banach space $X$ is symmetric if it has a symmetric basis, that is, a basis $\left\{x_{1}, x_{2}, \ldots, x_{n}\right\}$ such that:

$$
\left\|\sum_{k=1}^{n}\left|\alpha_{k}\right| x_{k}\right\|=\left\|\sum_{k=1}^{n} \alpha_{\pi(k)} x_{k}\right\|
$$

for any scalars $\alpha_{1}, \ldots, \alpha_{n}$ and any permutation $\pi$ of $\{1,2, \ldots, n\}$. This notion of symmetry is generalised by the following: An $n$-dimensional real Banach space $X$ is said to have enough symmetries (e.s.) (see [5]) if the only elements of $\mathcal{L}(X, X)$ which commute with every linear isometry of $X$ have the form $\kappa I$.

The (absolute) projection constant $\lambda(X)$ of $X$ is defined by:

$$
\lambda(X)=\sup \{\lambda(X, Y): X \subset Y\}
$$

where $\lambda(X, Y)$ is the (relative) projection constant of $X$ in $Y$, defined by:

$$
\lambda(X, Y)=\inf \{\|P\|: P \text { projects } Y \text { onto } X\} \text {. }
$$

\section{2-DIMENSIONAL SPACES, SELF-LENGTH}

Let $X$ be a 2-dimensional real Banach space, $S$ its unit sphere. We recall the definition of the self-length (or perimeter) $p(X)$ of $X$. Let $A$ be a convex polygon of vertices $\left\{a_{1}, a_{2}, \ldots, a_{n}\right\}$ inscribed in $S$, then (setting $a_{n+1}=a_{1}$ )

$$
p(A)=\sum_{k=1}^{n}\left\|a_{k+1}-a_{k}\right\|_{X}
$$

Received 21st March, 1995

Copyright Clearance Centre, Inc. Serial-fee code: 0004-9729/96 \$A2.00+0.00. 
is the "length" (with respect to the metric of $X$ ) of the polygon. Parallel to the classic definition of length of a curve we have the definition of self-length:

$$
p(X)=\sup \{p(A): A \text { a convex polygon inscribed in } S\} .
$$

It is clear that if $X$ is isometric to $Y(X \simeq Y)$ then $p(X)=p(Y)$. We list now some well known facts about self-length. For more detailed information we refer to [4]. $6 \leqslant p(X) \leqslant 8 ; p(X)=6$ if and only if $X \simeq H$, the affine regular hexagon; $p(X)=8$ if and only if $X \simeq l_{\infty}(2)$, the parallelogram. Of course, if $X \simeq l_{2}(2)$, then $p(X)=2 \pi$. Also $p(X)=p\left(X^{*}\right)$, where $X^{*}$ is the dual of $X . p(X)$ has been computed for the affine regular polygons and also for other spaces; see [4].

For the projection constants of 2-dimensional spaces we have: $\lambda(X)=\lambda\left(X^{*}\right) ; 1 \leqslant$ $\lambda(X) \leqslant 4 / 3 ; \lambda(X)=4 / 3$ if and only if $X \simeq H ; \lambda(X)=1$ if and only if $X \simeq l_{\infty}(2)$. The upper bound for $\lambda$ as well as the unicity statement about the hexagon is a difficult and important result recently proved in [7].

When the dimension is 2 the symmetry conditions become very simple. If $X$ is symmetric then there is a convenient basis such that in the representation of $X$ in $R^{2}$, the unit half sphere is symmetric with respect to the $x$-axis and the unit quarter sphere is symmetric with respect to the $(y=x)$-axis.

If $X$ is a (e.s.) space then the self-length and the projection constant satisfy the equation

$$
p(X)=8 / \lambda(X)
$$

(See [4]). This equality does not hold, however, for general spaces.

\section{MAIN RESULT}

We state here our main result:

Theorem 1. Assume that $X$ is a 2-dimensional real symmetric Banach space. Then $p(X) \geqslant 2 \pi$ and $p(X)=2 \pi$ if and only if $X \simeq l_{2}(2)$; consequently, $\lambda(X) \leqslant 4 / \pi$, and $\lambda(X)=4 / \pi$ if and only if $X \simeq l_{2}(2)$

We note that for spaces with (e.s) this result is not true in general since for $H$, which has (e.s.), we have $p(H)=6$ and $\lambda(H)=4 / 3$. Before proving the theorem we need some preliminary lemmas.

\section{Preliminary lemmas}

It is well known that every 2-dimensional Banach space $X$ is embeddable (linearly and isometrically) in a $L_{1}$ space, say $L_{1}[-\pi / 2, \pi / 2]$. A simple standard way of doing it was shown by Yost [10] (see also [8]): let $(x(t), y(t)),-\pi / 2 \leqslant t \leqslant \pi / 2$, be a 
parameterisation of half the unit sphere of $X$ (in a representation in $R^{2}$ ); then (the derivatives $x^{\prime}(t), y^{\prime}(t)$ exist almost everywhere and are in $\left.L_{1}[-\pi / 2, \pi / 2]\right)$ the subspace $\left[x^{\prime}, y^{\prime}\right] \subset L_{1}[-\pi / 2, \pi / 2]$ is isometric to $X^{*}$, the dual of $X$.

Lемма 1. Every 2-dimensional symmetric space $X$ is isometric to a subspace $V \subset L_{1}[-\pi / 2, \pi / 2]$ of the form $V=[r(t) \cos t, r(t) \sin t]$ with $r \geqslant 0 ; r(-t)=$ $r(t) ; r(\pi / 2-\tau)=r(\tau), 0 \leqslant \tau \leqslant \pi / 2$.

PROOF: We can choose a symmetric basis so that in the representation in $R^{2}$ we have a parameterisation $P(t)=(x(t), y(t))$ of the unit sphere $C$ such that

$$
\begin{gathered}
x( \pm \pi / 2)=0 ; x(0)=1 ; x(-t)=x(t) ; x^{\prime}(t) \leqslant 0,0 \leqslant t \leqslant \pi / 2 \\
y( \pm \pi / 2)= \pm 1 ; y(0)=0 ; y(-t)=-y(t) ; y^{\prime}(t) \geqslant 0,0 \leqslant|t| \leqslant \pi / 2 \\
x(\pi / 2-t)=y(t) ; y(\pi / 2-t)=x(t), 0 \leqslant t \leqslant \pi / 2
\end{gathered}
$$

If $Q(t)=(y(t),-x(t))$, then $Q$ is also a parameterisation of $C$, and therefore $\left[y^{\prime},-x^{\prime}\right] \subset L_{1}[-\pi / 2, \pi / 2]$ is isometric to $X^{*}$. Now $\left(y^{\prime}(t),-x^{\prime}(t)\right)$ is in the same octant as $(\cos t, \sin t)$; so, by considering for example only the first octant, there is a rearrangement $t \rightarrow \phi(t)(\phi(0)=0 ; \phi(\pi / 4)=\pi / 4)$ and a positive $L_{1}$-function $r(t)$ such that almost everywhere in $[-\pi / 2, \pi / 2]$ we have

$$
\left(y^{\prime}[\phi(t)],-x^{\prime}[\phi(t)]\right)=(r(t) \cos t, r(t) \sin t) .
$$

Finally recall that if $X$ is symmetric then also $X^{*}$ is symmetric; therefore the family of duals of symmetric spaces coincides with the family of all symmetric spaces.

EXAMPLE. If $X$ is 2-dimensional real euclidean space, then note that we can take $(x(t), y(t))=(\cos t, \sin t), \phi(t)=t$, and $r(t)=1$ in Lemma 1 and its proof.

Lemma 2. $[2,8]$ If $V$ is a 2-dimensional real space and $V \subset L^{1}$, then $\lambda\left(V, L^{1}\right)=$ $\lambda(V)$.

REMARK. It is a well known fact that if $V$ is isometric to $W$ then $\lambda(V)=\lambda(W)$. For 2-dimensional real spaces with (e.s.), this fact follows immediately also from (1).

LEMMA 3. Let $r$ be an element of $L_{1}[-\pi / 2, \pi / 2]$ such that:

$$
r(t) \geqslant 0 ; r(-t)=r(t) ; r(\pi / 2-\tau)=r(\tau)(\tau \in[0, \pi / 2]) .
$$

Then, if

$$
\sigma(t)=\int_{-\pi / 2}^{\pi / 2}|\cos (\alpha-t)| r(\alpha) d \alpha
$$

we have

$$
\sigma(t)=\sigma(-t) ; \sigma(\pi / 2-t)=\sigma(t)
$$


Proof: The first equality follows from the fact that

$$
\sigma(t)=\int_{0}^{\pi / 2}(|\cos (\alpha+t)|+|\cos (\alpha-t)|) r(\alpha) d \alpha .
$$

With the change of variable $\alpha=\pi / 2-\beta$ we obtain

$$
\begin{aligned}
\sigma(t) & =\int_{0}^{\pi / 2}(|\cos (\pi / 2-\beta+t)|+|\cos (\pi / 2-\beta-t)|) r(\beta) d \beta, \\
\sigma(\pi / 2-t) & =\int_{0}^{\pi / 2}(|\cos (\pi-\beta-t)|+|\cos (-\beta+t)|) r(\beta) d \beta=\sigma(t) .
\end{aligned}
$$

Lemma 4. Let $r$ and $\sigma$ be as in Lemma 3 and set $V=\left[v_{1}, v_{2}\right] \subset L_{1} ; U=$ $\left[u_{1}, u_{2}\right] \subset L_{\infty} ; v_{1}=r(t) \cos t ; v_{2}=r(t) \sin t ; u_{1}=s(t) \cos t ; u_{2}=s(t) \sin t ; s(t)=$ $c /(\sigma(t)) ; 1 / c=\int_{0}^{\pi / 2}(r(t)) /(\sigma(t)) d t$. Then, if we define $P: L_{1} \rightarrow V$ by $P=u_{1} \otimes v_{1}+$ $u_{2} \otimes v_{2}$, the operator $P$ is a projection onto $V$ with $\|P\|=c$.

ProOF: We must show that $\left\langle u_{i}, v_{j}\right\rangle=\delta_{i j}$. Note that by (2) we have $s(t)=$ $s(-t) ; s(\pi / 2-t)=s(t)$. We have

$$
<u_{1}, v_{2}>=<u_{2}, v_{1}>=\int_{-\pi / 2}^{\pi / 2} r(t) s(t) \cos t \sin t d t
$$

which is 0 since the integrand is an odd function. Moreover

$$
<u_{1}, v_{1}>=2 \int_{0}^{\pi / 2} r(t) s(t) \cos ^{2} t d t=2 \int_{0}^{\pi / 2} r(t) s(t) \sin ^{2} t d t=<u_{2}, v_{2}>
$$

thus

$$
<u_{i}, v_{i}>=\int_{0}^{\pi / 2} r(t) s(t) d t=c \int_{0}^{\pi / 2} \frac{r(t)}{\sigma(t)} d t=1
$$

Recall now that the Lebesgue function $\Lambda$ of the operator $P$ is defined by $\Lambda(\phi)=$ $\int_{-\pi / 2}^{\pi / 2}\left|u_{1}(\phi) v_{1}(t)+u_{2}(\phi) v_{2}(t)\right| d t$ and that the norm of $P$ is given by $\sup \{\Lambda(\phi): \phi \in$ $[-\pi / 2, \pi / 2]\}$, see for example, [1] and [3]. As we shall see in our case, the Lebesgue function is constantly equal to $c$. Indeed we have

$$
\Lambda(\phi)=s(\phi) \int_{-\pi / 2}^{\pi / 2} r(t)|\cos (\phi-t)| d t=s(\phi) \sigma(\phi)=s(\phi) \frac{c}{s(\phi)}=c .
$$

We shall prove that $\|P\| \leqslant 4 / \pi$. Once this is done, since by Lemma $2 \lambda(V) \leqslant\|P\|$, recalling (1) we obtain that

$$
p(V)=\frac{8}{\lambda(V)} \geqslant 2 \pi
$$

Since $1 /(\|P\|)=\int_{0}^{\pi / 2}(r(t)) /(\sigma(t)) d t=J$, we have to show that $J \geqslant \pi / 4$. 
LEMMA 5. J can be written in the form

$$
J=\int_{0}^{\pi / 4} \frac{r(t) d t}{(\cos t+\sin t) \int_{0}^{\pi / 4} \cos \alpha r(\alpha) d \alpha+\int_{t}^{\pi / 4} \sin (\alpha-t) r(\alpha) d \alpha} .
$$

Proof: First it is clear that $J=2 \int_{0}^{\pi / 4}(r(t)) /(\sigma(t)) d t$ and it is also easy to see that $\sigma(t)=\int_{0}^{\pi / 4}(|\cos (\alpha+t)|+|\cos (\alpha-t)|+|\sin (\alpha-t)|+|\sin (\alpha+t)|) r(\alpha) d \alpha$. Since $0 \leqslant \alpha \leqslant \pi / 4$ and $0 \leqslant t \leqslant \pi / 4$ we have:

$$
\sigma(t)=\int_{0}^{\pi / 4}(2 \cos \alpha \cos t+\sin \alpha \cos t+\cos \alpha \sin t) r(\alpha) d \alpha+\int_{0}^{\pi / 4}|\sin (\alpha-t)| r(\alpha) d \alpha
$$

where

$$
\int_{0}^{\pi / 4}|\sin (\alpha-t)| r(\alpha) d \alpha=\int_{0}^{\pi / 4} \sin (t-\alpha) r(\alpha) d \alpha+2 \int_{t}^{\pi / 4} \sin (\alpha-t) r(\alpha) d \alpha .
$$

Thus we obtain

$$
\sigma(t)=2 \int_{0}^{\pi / 4}(\cos \alpha \cos t+\cos \alpha \sin t) r(\alpha) d \alpha+2 \int_{t}^{\pi / 4} \sin (\alpha-t) r(\alpha) d \alpha
$$

from which the formula for $J$ follows.

\section{Proof of Theorem 1}

Pointing out the dependence of $J$ on the function $r$, we shall write

$$
\begin{gathered}
J=J(r)=\int_{0}^{\pi / 4} \frac{r(t)}{\delta_{r}(t)} d t \\
\delta_{r}(t)=(\cos t+\sin t) \int_{0}^{\pi / 4} \cos \alpha r(\alpha) d \alpha+\int_{t}^{\pi / 4} \sin (\alpha-t) r(\alpha) d \alpha .
\end{gathered}
$$

Let $A=\left\{r \in L_{1}[0, \pi / 4]: r \geqslant 0\right\}$; we first prove that

$$
\inf \{J(r), r \in A\}=\pi / 4 .
$$

We have (omitting the index $r$ in the functional $\delta$ ):

$$
\begin{aligned}
& \delta^{\prime}(t)=(\cos t-\sin t) \int_{0}^{\pi / 4} \cos \alpha r(\alpha) d \alpha-\int_{t}^{\pi / 4} \cos (\alpha-t) r(\alpha) d \alpha \\
& \delta^{\prime \prime}(t)=-(\cos t+\sin t) \int_{0}^{\pi / 4} \cos \alpha r(\alpha) d \alpha+r(t)-\int_{t}^{\pi / 4} \sin (\alpha-t) r(\alpha) d \alpha
\end{aligned}
$$


and consequently

$$
\delta^{\prime \prime}+\delta=r ; \delta(0)>0 ; \delta^{\prime}(0)=0 ; \delta^{\prime}(\pi / 4)=0
$$

These imply that

$$
J(r)=\frac{\pi}{4}+\int_{0}^{\pi / 4} \frac{\delta^{\prime \prime}}{\delta} d t
$$

But we have

$$
\int_{0}^{\pi / 4} \frac{\delta^{\prime \prime}}{\delta} d t=\left[\frac{\delta^{\prime}}{\delta}\right]_{0}^{\pi / 4}+\int_{0}^{\pi / 4}\left(\frac{\delta^{\prime}}{\delta}\right)^{2} d t
$$

and hence we get

$$
J(r)=\frac{\pi}{4}+\int_{0}^{\pi / 4}\left(\frac{\delta^{\prime}}{\delta}\right)^{2} d t \geqslant \frac{\pi}{4} .
$$

It is clear that every $r=$ constant (positive) is a minimum point for $J$ on $A$; also $J(r)=\pi / 4 \Leftrightarrow \delta^{\prime}=0$. We show now that constants are the only minimum points. If the function $r$ is a point of minimality, it follows that $\delta^{\prime}=\delta^{\prime \prime}=\dot{0}$, and this implies that $\delta$ is constant and (since $\delta^{\prime \prime}+\delta=r$ ) that $r=$ constant.

\section{REMARK}

It is well known (see for example, [6]) that the value of the projection constant of $n$-dimensional euclidean space is

$$
\lambda\left(l_{2}(n)\right)=\frac{n \Gamma\left(\frac{n}{2}\right)}{\sqrt{\pi} \Gamma\left(\frac{n+1}{2}\right)} .
$$

In view of the second statement in Theorem 1 , one could ask whether it is true, also for $n>2$, that for $n$-dimensional symmetric spaces $X_{n}$ one has $\lambda\left(X_{n}\right) \leqslant \lambda\left(l_{2}(n)\right)$. The answer is no even for $n=3$ as is shown in the example constructed by Positselskii in [9]. In fact he has computed for every $n$ the exact value $K_{n}$ of the absolute projection constant of a special sequence of symmetric spaces (Marcinkiewicz spaces); it turns out that $K_{n}>\lambda\left(l_{2}(n)\right)$ for all $n>2$ but $n=4$.

\section{NOTE}

After completing this work we were informed that the result $\lambda(X) \leqslant 4 / \pi$ has been proved (independently and with totally different method) in: "Projections onto symmetric spaces" by Hermann Koenig, to appear in Quaest. Math. 


\section{REFERENCES}

[1] B.L. Chalmers and F.T. Metcalf, 'The determination of minimal projections and extensions in $L^{1}$, Trans. Amer. Math. Soc. 329 (1992), 289-305.

[2] B.L. Chalmers and F.T. Metcalf, 'A simple formula showing $L^{1}$ is a maximal overspace for two-dimensional real spaces', Ann. Polon. Math. 56 (1992), 303-309.

[3] C. Franchetti and E.W. Cheney, 'Minimal projections in $L^{1}$-space', Duke Math. J. 43 (1976), 501-510.

[4] C. Franchetti and G.F. Votruba, 'Perimeter, Macphail number and projection constant in Minkowski planes', Boll. Un. Mat. Ital. B 13 (1976), 560-573.

[5] D.J.H. Garling and Y. Gordon, 'Relations between some constants associated with finite dimensional Banach spaces', Israel J. Math. 9 (1971), 346-361.

[6] B. Grünbaum,, 'Projection constants', Trans. Amer. Math. Soc. 95 (1960), 451-465.

[7] H. Koenig and N. Tomczak-Jaegermann, 'Norms of minimal projections', J. Funct. Anal. 119 (1994), 253-280.

[8] J. Lindenstrauss, 'On the extension of operators with a finite-dimensional range', Ilinois J. Math. 8 (1964), 488-499.

[9] E.D. Positselskii, 'Projection constants of symmetric spaces', Math. Notes 15 (1974), 430-435 (Translated from Mat. Zametki 14 (1974), 719-727).

[10] D. Yost, ' $L_{1}$ contains every two-dimensional normed space', Ann. Polon. Math. 49 (1988), 17-19.

Department of Mathematics

University of California

Riverside CA 92521

United States of America
Departmento di Matematica Applicata

Università di Firenze

50139 Firenze

Italy 